\title{
Rapid lung magnetic resonance imaging in children with pulmonary infection: reply to Sodhi et al
}

\author{
H. Nursun Ozcan ${ }^{1} \cdot$ Ayșegul Gormez ${ }^{1}$ - Yasemin Ozsurekci ${ }^{2} \cdot$ Jale Karakaya $^{3}$ • \\ Berna Oguz $^{1}$ - Sule Unal ${ }^{4}$ - Mualla Cetin ${ }^{4} \cdot$ Mehmet Ceyhan $^{2} \cdot$ Mithat Haliloglu $^{1}$
}

Received: 1 February 2017 / Accepted: 15 February 2017 / Published online: 7 March 2017

(C) Springer-Verlag Berlin Heidelberg 2017

Dear Editor,

We thank Sodhi et al. [1] for their interest in and comments on our article "Magnetic resonance imaging of pulmonary infection in immunocompromised children: comparison with multidetector computed tomography" [2].

We agree that with the advancements in MRI technology and new lung MR techniques, such as ultrashort echo time, utility of lung MRI will further improve the detection rate for various lung lesions. As referenced in our recent article [2], using both radial $\mathrm{k}$-space techniques and single-shot halfFourier sequences, a high correlation with $\mathrm{CT}$ and high sensitivity may be achieved. Since it does not entail any radiation and can be performed fast enough, MRI may be a viable option in the follow-up of immunocompromised children. As we have both noted, some patients still have high risk for radiation including those with DNA repairing deficiency syndromes (ataxia telangiectasia, Bloom syndrome, Fanconi anemia), in which case, lung MRI may be used.

Mithat Haliloglu

mithath@hacettepe.edu.tr

1 Department of Radiology, Division of Pediatric Radiology,

Hacettepe University School of Medicine,

Sihhiye 06100 Ankara, Turkey

2 Department of Pediatric Infectious Disease, Hacettepe University School of Medicine, Ankara, Turkey

3 Department of Biostatistics, Hacettepe University School of Medicine, Ankara, Turkey

4 Department of Pediatric Hematology, Hacettepe University School of Medicine, Ankara, Turkey
One of our goals was to compare various MR sequences commonly used in lung MRI. We demonstrated that fatsaturated, periodically rotated, overlapping parallel lines with enhanced reconstruction sequences have higher sensitivity in the detection of pulmonary abnormalities, particularly nodules smaller than $5 \mathrm{~mm}$. This technique and the single-shot half-Fourier sequence achieved the same sensitivities in detecting ground-glass opacities and nodules larger than $5 \mathrm{~mm}$.

On the basis of our findings, we believe that lung MRI is promising for detection of pulmonary abnormalities in immunocompromised children with clinically suspected pulmonary infections. We think that lung MRI can be used much more commonly both in immunocompetent and immunocompromised children for the detection of lung lesions.

Compliance with ethical standards

Conflicts of interest None

\section{References}

1. Sodhi KS, Bhatia A, Khandelwal N, Rapid lung magnetic resonance imaging in children with pulmonary infection. Pediatr Radiol doi 10.1007/s00247-017-3811-3

2. Ozcan HN, Gormez A, Ozsurekci Y et al (2017) Magnetic resonance imaging of pulmonary infection in immunocompromised children: comparison with multidetector computed tomography. Pediatr Radiol 47:146-153 